\title{
Using Technology-Assisted Learning to Transform Employee Development
}

\author{
Eugene J. Monaco \\ University at Albany, USA
}

\begin{abstract}
This paper explores the implications for $e$ learning delivery of workplace education in the U.S., as demonstrated in the New York State government's scaling of training to reach its 159,000 employees through an e-learning curriculum project in partnership with Rockefeller College of the University at Albany, State University of New York. By tracking the evolution of e-learning in workplace education from early introductions in the private sector to migration into the public sector, the author illustrates that successful transformation is achieved when projects like this substantiate the proof of concept that mediated learning delivery brings both a return on investment and multiple advantages over traditional classroom education for both employee and employer. The project evaluation data further substantiate the conclusion that e-learning is rapidly becoming a widespread delivery method of choice for continuing professional education in the future U.S. workplace.
\end{abstract}

\section{Introduction}

The significance of e-learning and knowledge management in the workplace cannot be overstated. In today's technology-driven working environments the accelerated pace of change is a constant, and organizations are pressed to rapidly train and retrain workers in new technologies, products, and services with increasingly limited timelines and resources. The constant and unrelenting need to manage the organizational knowledge base to keep it current and readily accessible to all stakeholders in the work environment is an additional factor driving the need for just-in-time training and retraining of the workforce at all levels. Forward thinking employers have recognized e- learning as more productive from both the employers' and the employees' perspectives. They acknowledge that e-learning increases the possibilities for how, where, and when employees can engage in training. In the public sector integrating e-learning into the workplace has been much slower to adapt as a training option but in recent years this reluctance has changed considerably.

\section{The Case for Advancing E-Learning}

In the United States today, technology-based training has continued a rapid expansion as it has become easier for developers and end users to master. As a training medium e-learning has gained substantial traction over the past decade and is quickly becoming a preferred delivery mode in workplace learning settings across organizations of various sectors and sizes. Since 2000, a variety of online training methodologies have emerged as well as different types of training such as web-based training, live streaming audio and video, and the use of message boards and chat rooms. As early as 2001 e-learning was described as the next "killer application" [5]. The American Society for Training and Development (ASTD) further substantiates this fact in its most recent industry report that documents that using technology to deliver training is increasing in popularity, and in 2011 there was a considerable increase in the use of self-paced non- network computer learning from 1.8 percent in 2010 to 8.5 percent in 2011 in the use of technology both synchronous and asynchronous will continue to grow in the coming years [11].

At the same time, it is interesting to note is the rapid advancement of e-learning as an increasingly widespread tool used for workplace training by organizations has far outpaced research findings in this area. The overall impact of this accelerated use of e-learning is still in the formative stage and impact evaluations are now becoming more prevalent. Plainly, activity in this area has continued to outpace academic research on the topic [22]. Therefore a word of caution should be rendered regarding the overall impact of its effectiveness in the training world. It may take some time for the academic world to catch up with what is happening; nevertheless, there is no way to corral the momentum that is being unleashed by the e-learning movement. Its continued growth and increasing acceptance can be traced to the many advantages it provides to both employers and employees. From the learners' perspectives, elearning offers increasingly greater amounts of control over their own learning such as self-pacing and the freedom to enter and leave instructional material as they desire. From the employers' points of view, it offers a number of advantages such as scalability, conformity of message, and potential cost savings. At the same time, educators and researchers 
must remain mindful of the impending danger of moving too fast without producing proper validation through conducting structured impact and process studies to gage overall effectiveness of on-line education training programs.

As e-learning has so rapidly progressed, it has also generated a number of critical challenges which are a real concern for e-learning developers and their accompanying content experts. First and foremost, developers and content experts must find ways to keep learners engaged with the content. A recent study Going the Distance: Online Education in the United States, 2011 address the concern over high dropout rates and lower content retention exhibited by online learners in comparison to traditional learning which has led to questions regarding quality, value, and impact of online offerings [14]. As a result, efforts are underway to focus attention on increasing the motivation and involvement of learners through a variety of tools adopted from the video game world, such as training games, which can be used in the presentation and practice of training topics. This has also led to the increasing use of usability testing (UT) with the intention of improving educational experiences, learner outcome, and retention rates. Long used by web developers to assess and improve web sites and applications for ecommerce and other business uses, UT has been gaining traction for evaluating e-learning effectiveness [13]. Recent studies have pointed to usability as having the potential to significantly improve courses for student learning and retention [1]. They state that poorly developed e-learning platforms disturb accommodation of new concepts in addition to impairing overall retention. They further assert that UT provides opportunity for course developers to gain insight into how their learners engage so the developers can discover ways to avoid the learner confusion, frustration and distraction that impedes learning and leads to student dropout [1]. So, as current authoring and learning management systems, as well as tools such as animation software, video streaming, and surveying and testing, lead developers to deliver more on-line courses in abbreviated timeframes, the challenge is to ensure quality and effectiveness. UT is one such answer; it needs to be given careful consideration and is strongly recommended as a means to ensure the development of usable content by learners.

Although employers recognize the ways in which e-learning expands employees' learning and helps meet organizational needs, they are also aware of the barriers to its use. These include the high cost of developing and purchasing e-learning programs, the lack of employee's available time to devote to workplace learning, and the appropriateness of currently available learning content.
Organizations that have been successful in implementing e-learning programs have found ways to overcome the barriers. These include:

- $\quad$ Building a learning culture;

- $\quad$ Conducting extensive research and planning through testing and piloting in limited implementations before scaling;

- Communicating the value of e-learning across the organization; and

- Integrating it into their knowledge management systems [4].

Employers have seen that successful e-learning programs provide the opportunity to deploy just-intime training and reduce the time span between learning and its transfer to work. Employees see the advantages as greater convenience and flexibility accompanied by greater control over their own learning and their own pace of learning.

Over the last three centuries as we progressed from the Industrial Age of the 19th century through the Information Age of the 20th century and into the Knowledge Age of the 21st century, the need for learning in the workplace has become more defined, and the learning environment has changed from the one-on-one apprentice method of teaching the skills of a trade, to the formal classrooms of the 20th century and finally to electronically delivered training in the 21st century. As early as 2002 future applications for online learning were predicted, three of which have taken hold:

1. The increased interest in and use of mobile learning, particularly in developing countries where access to laptops and computers is limited;

2. The rapid proliferation of e-books, which has promoted more interest in online exploration;

3 . The steady growth of global virtual universities since the beginning of the 21 st century [8].

A recent survey, the Global Internet User Survey of 2012, which is consider a highly trusted independent source for Internet policy and future development, reported that creating a more highly educated global workforce through online learning was significantly important to $56 \%$ of those surveyed [7]. The steady migration to e-learning in the global work environment is clear.

In the United States several observations can be made. Foremost, technology-based training has continued a rapid expansion, closely mirroring the global movement, and reaching back to the early 2000's when U.S. corporations realized the economic savings resulting from converting their traditional training delivery to e-learning. For example, IBM reported saving over \$200 million in 1999 by converting to e-learning, and Ernst and Young reduced costs by 35 percent while improving scalability and consistency [18]. Forester Research, an independent research firm that helps companies assess the effect of technology change on their operations recently examined the way online 
learning was earning companies a positive return on investment (ROI) in less than a year. Forester found that companies whose businesses were spread across many locations could make good business decisions to implement an online learning program as a replacement for face-to-face learning and as a complement to other instructor-led training through blended learning. Whether employees took compliance training, desktop skills development, or leadership training, online learning was more flexible, consistent, and repeatable with minimal travel costs. Forester's findings showed a $69 \%$ ROI for these approaches to employee learning [3].

These studies reveal compelling reasons to turn to e- learning for training, but developing simple to use e- learning programs has not been easy recently.

The impact that easy-to-use authoring tools made changed the dynamics of developing and producing e-learning programs. It has become increasingly easier for content course developers to master the intricacies of developing e-learning programs without relying on sophisticated computer programming help or technical support because of user-friendly authoring programs like Lectora and Articulate. E-learning has continued to grow in popularity as a choice for training and as indicated is rapidly gaining momentum as a favored choice to conduct workplace training for employees. Therefore, it's not surprising to observe in the American Society for Training \& Development's (ASTD) 2012 State of the Industry reporting that technology-based training accounted for over $37 \%$ of all workplace training offered by organizations in the U.S. in 2011 [11]. If the trend of using e-learning as a training medium continues on this upward vortex, it will easily become the preferred delivery mode in workplace learning settings in organizations of various sectors and sizes in the 21 st century. In looking back signs of its growth was beginning to take hold. As early as 2000, e-learning began to develop as a well-accepted teaching and learning tool. As a consequence, a variety of online training methodologies have emerged as well as different types of training such as web-based training, live streaming audio and video, and the use of message boards and chat rooms with social media. It's taken a while to take hold but the data is clearly trending in the direction of using technology assisted instruction to support training. ASTD's reporting data is a very strong indication of how rapidly the training industry has moved away from the traditional classroom delivery to a more robust and versatile e-learning approach. The conclusion is that the use of technology for both synchronous and asynchronous learning will continue to gain traction and acceptance in the training world as its advantages over traditional classroom training will become more apparent.

\section{Government Adaptation of E-Learning for the Workplace}

Over the past decade the trend in the United States has also been for government at the local, state, and national levels to follow the corporate model to use online learning as a more cost- effective solution to workplace training. The increasing demand on and expectations for governments as well as the financial constraints in today's economic climate further drive more cost effective workplace training approaches to be used in the public sector.

In addition to the economic benefits, similar advantages that the private sector has experienced such as convenience, standardization of content and self-service delivery have also made e-learning highly desirable for government agency workforce training. In a knowledge and information society elearning in the workplace has great potential to transform how and when employees learn as well as how to effectively transfer learning to performance. Studies conducted by the U.S. Department of Education and the U.S. Army Research Institute for Behavioral and Social Sciences both demonstrated that online learning was also more likely to meet instructional objectives and lead to desired learning outcomes when compared to classroom delivery [19] [20]. The results of these studies have provided government with much needed empirical evidence regarding the efficacy of moving in the direction of supporting e-learning as an effective way to offer training for employees. Overall the public sector has been much more cautious and deliberate in moving towards endorsing technology based training but given these recent developments, the tide is now quickly shifting.

\section{New York State Government's Migration to E-Learning}

A case in point is to examine the migration to elearning for the public workforce of the State of New York. In order to better understand how e- learning emerged as a highly desired delivery medium for training of the public sector workforce in New York State, a closer examination of how state government works is necessary. In New York, the state government is a large and complex bureaucracy employing over 159,000 workers. These workers are not all in a central location but are distributed over some 69 different agencies that are highly diverse, managing the business of the state, including infrastructure, transportation, social and health care services, regulations, recreation, correctional services, juvenile justice, environmental conservation, tax and finance. To complicate matters the state workforce is a diverse blend of rural, suburban, and urban populations. Managing and 
training the diverse workforce to be effective in carrying out the wide range of duties and responsibilities that employees have is both complex and a monumental task to accomplish. The fundamental charge for all the state government entities is to provide goods and services to over 19 million state citizens efficiently and effectively. Each agency in the state system possesses unique skill and knowledge requirements that can be directly related to the various job positions as well as associated occupational training requirements. Many of the knowledge and skill requirements are specific to the work that the agency does, so the agencies have defined internal training needs and have over time developed specific training and development programs to meet those unique needs. Throughout the state system training has become quite insular in many state agencies and resistance to common core training has arisen over time.

Intriguingly, but not unusual, is that the one common theme over time that spanned these groups of employees in all agency training and development programming was management and supervisory training and development, which is logical considering the number of supervisors and managers inherently needed to manage a workforce the size of that of New York State government. In fact, the most current statistics obtained from the New York State Department of Civil Service reports more than 15,000 experienced and new supervisory personnel are in state government service at any given time [14]. To succeed in keeping this government working well, these managers and supervisors require a working knowledge and understating of supervisory principles at all levels regardless of experience. It is also a common requirement that all supervisors regardless of their agency affiliation be responsible for planning, organizing, directing, and controlling the activities of employees they are supervising whose job it is to perform the necessary tasks and services of the agency's work [17]. There is a strong and ongoing need to provide adequate and appropriate supervisory training and development in these following areas:

- Delegation: Trusting another person to do a job for which you remain responsible

- Leadership: The ability to influence the actions of others

- Decision Making: Choosing actions from available alternatives to achieve results

- Team Building: Helping groups achieve common goals through interaction and unity

- Communication: The transmission of a message between a sender and a receiver

- Counseling: Correcting unacceptable employee behavior

- Evaluating Performance: Letting people know where they stand
- Managing Diversity: Accepting, incorporating and empowering diverse human talent

- Motivation: Providing incentives for action

Keeping this information relevant, updated and easy to use, New York State government embarked on a major reorganization effort to achieve more overall efficiencies in managing its workforce. In an effort to achieve this goal of making state government more efficient and streamlined a number of recommendations emerged around better organizing various functions of state government through introducing a series of consolidation initiatives of common functions such as human resources, purchasing, computer systems, and training [12]. Conditions were, therefore, prime for considering alternative methods to meeting the need across state agencies to conduct not only supervisory training consistent in content and quality to address common supervisory principles and practices but also other key common core functional areas as well. What transpired to aid and make this possible was an interesting and ongoing arraignment between a university-based continuing professional education program proficient in curriculum design and development in the e-learning environment and the state government entity responsible for implementing such massive reform efforts. The university based program was able to effectively assist a large state government bureaucracy to successfully develop a series of e-learning courses in the area of supervisory management as well as other workplace topics that addressed these common concerns and issues. Given the implications and impact of this massive government reform, it was important that employees be well trained to provide the appropriate response and meet the needs of the citizens they serve in their corresponding areas regardless of the products or services being delivered. Since this was not always the case, conditions were prime to consider alternate methods to meet these ends. One area that stood out as being in need of immediate improvement was training for employees across the agencies in a more consolidated, standard and modular way. Historically, individual state agencies had assumed the responsibility for training and developing their own workforces. Such an approach was necessary and understandable for certain agency-specific skillbased jobs. However, on a wider level and over time agency-directed training was unproductive in many respects, leading to undesirable and uneven results on a statewide basis. To change this restricted and largely unproductive approach, the State of New York's Governor's Office of Employee Relations (GOER), which has the responsibility of overseeing all training and development for New York State employees, reached out to the University at Albany's Professional Development Program (PDP) at Rockefeller College as a resource to develop online 
learning courses to deliver not only to supervisors, but also to all 159,000 employees in a standard and consistent format and at a more professional level.

GOER requested that PDP develop a comprehensive proposal to provide updated, just-in- time courses for supervisory training as well as work on the development of six other courses that were crossfunctional in nature and that required consistent messaging and information to be delivered across the entire state workforce. The six courses identified to be developed were: (1) Equal Employment Opportunities, (2) Sexual Harassment in the Workplace, (3) Providing Reasonable Workplace Accommodations, (4) Performance Appraisal, (5) Attendance and Leave Benefits, and (6) Knowledge Management. These initial courses were to be the first in a series of ten e-learning courses dealing with other work-related performance topics which would result in workers obtaining consistent and uniform messaging delivered statewide. The four remaining courses are still under discussion.

\section{E-Learning Courses for State Government}

As a leading-edge, university-based training organization, PDP was aware of the potential for technology to be the successful solution for this workforce education and training. However, they were also aware of the many inherent challenges in implementing a program of this magnitude in a short timeframe.

For over 37 years PDP had been providing a comprehensive continuing professional education program for public sector employees in New York State government. PDP was a provider of training for the New York State workforce. In its formative years its training was manly classroom based. However, PDP had been creating e-learning for New York State government agencies as well for over 15 years. It had been one of the first in the United States to begin to offer technology-delivered programs for state employees and had documented success in its programming. Its approach to offering online learning evolved and became more sophisticated as government realized a need for and willingness to embrace online training.

\section{Early E-Learning Experiences}

PDP's initial e-learning program in 1998 involved courses for real property real estate assessors in New York State, culminating in a mandatory comprehensive exam, which was administered onsite due to concerns of verifying the identity of the test-takers. An early Learning Management System (LMS) was selected to administer the program, which was a fairly popular product at the time--Top Class. Although the turnkey system had advantages, such as integration of discussion tools and the ability to offer courses to specific cohorts of trainees, it proved expensive to maintain and incorporated many advanced features that the agency did not need.

Based on that experience, PDP began developing e-learning management systems tailored directly to meeting agency needs for trainee registration, tracking and evaluation with great success. This proprietary approach allowed PDP a great deal of flexibility in course design, including dynamic database-driven courses that responded to learner input as well as the ability to build content without relying on standard authoring systems that had limiting features for the expression of content.

For a workforce that approached web-based training with a great deal of hesitance and skepticism, due to previous experience with unsophisticated courses of page after page of text followed by multiple choice questions, PDP was challenged to demonstrate that e-learning could be both engaging and effective. An early project in 2001 converting classroom training for food stamps eligibility workers in the state of Vermont resulted in an online program so effective that in a single year Vermont went from having one of the highest error rates in the nation to one of the lowest. The consequence of this success directly led to other elearning "converts" who wanted more online training and looked to PDP to take the lead with the production of interactive e-learning courses.

Many more successes followed, including creation of a suite of training modules for the New York State Department of Health's Tobacco Control Program in 2005. This program, which targeted substance abuse providers with knowledge and skills to reduce tobacco dependence in individuals with other dependencies, had been very successful, despite early predictions that its target audience would not have the technical skill, interest, or discipline to complete the 26 hours it offers. Faced with this challenge, PDP's development team chose a graphically rich, highly immersive approach to content presentation, using video and other media to illustrate and reinforce key concepts and skillbuilding and found time and again that learners respond positively to content that engages the senses and provides ample challenges and opportunities for interaction.

From these experiences PDP addressed the need to make e-learning programs more robust and interactive and established standards for the development of e-learning courses, which have been accepted by it sponsors and the university. 


\section{Establishing Standards for E-Learning Courses}

In 2012 PDP published a guide to best practices in e-learning design and uniformity in developing elearning courses across a range of different content areas. The guide drew upon principles for the design of multimedia instruction such as interactivity, coherence, modality, signaling, personalization, and redundancy that had been proved through research as successful. The guide also drew on the experience in e-learning design and development PDP had. Using this guide to author courses ensures that learners are able to navigate and complete a course without experiencing frustration and navigation problems. For those new to developing e-learning courses, the guidelines provide guidance in building wellconstructed, user-friendly courses.

The guide addresses topics that are essential to elearning course development:

- Style Guidelines

- Interface Design

- Accessibility Standards

The greatest challenge can be creating instructionally sound and engaging content for remote learners who cannot directly access each other or the instructor. Enabling their learning requires securing and keeping their attention and providing sound instruction and relevant practice. Over the past decade much research has been done into the way that people learn in a computer-based environment. The section on "Research-Based Principles for the Design of Multimedia Instruction" provides an introduction to some basic principles of good design.

\section{Addressing Sharable Content Reference Model (SCROM) Standards}

With the advent of enterprise Learning Management Systems (LMS) integration into state government training necessitated that an adoption of an off-the-shelf authoring tool that publishes SCORM-compliant courses be undertaken. SCORM is a set of technical standards for e-learning software products. SCORM tells programmers how to write their code so that it can "play well" with other elearning software. It is the de facto industry standard for e-learning interoperability. Specifically, SCORM governs how online learning content and LMS communicate with each other. SCORM does not speak to instructional design or any other pedagogical concern; it is merely a technical standard.

PDP chose to use, Trivantis Lectora, to meet SCORM compliant standards. This authoring system allows non-technical staff an entry into e- learning, however its library of programmed interactions was found to be somewhat limited, and there were other technical limitations as well. To enhance content, PDP's solution was to develop rich content in Adobe Flash and integrate these interactions and multimedia elements into Lectora. The suite of ten courses under development for the New York State Governor's Office of Employee Relations on a variety of topics, including mandated policy training and soft skills uses the Lectora authoring system with enhancements.

\section{Planning for E-Learning Delivery}

Utilizing the principles found in the guide for elearning, PDP began to build its courses. The initial step in the process is to identify and validate content; the next step is to build a design that would translate into an asynchronous e-learning platform that could be delivered as a SCORM package for installation on the New York State Learning Management System (SLMS). An overall design strategy would be proposed for each of the ten courses.

\section{Design Strategy}

The design strategy would be the same for each course, serving as a roadmap that would be followed as the design for each course developed. It would at the outset itemize the goals and objectives, outline the instructional design approach, and provide a basic content outline. The process would follow the Instructional Systems Design (ISD) model in performance-based training created by Clark and applied for both classroom and online delivery of training [2]. For illustrative purposes the following Attendance and Leave Benefits course map illustrates the way in which the process was used for building each course used in the series.

Table 1 provides how the course would be designed and structured. Content was designed to be presented as a series of pages with text, related graphics, and user interactions. The pages focus on interactions and limit material dissemination to brief overviews and agency-specific resource sheets. Interactions based on applied learning focus on allowing learners to practice their knowledge about each topic and apply it to a variety of scenarios. Feedback is integrated into the course to confirm correct practices and to provide guidance and feedback for incorrect practices. For example, using the Introduction of Attendance and Leave benefits as a model that all courses would follow is used here for illustrative purposes. The course overviews key information required as related to attendance and leave benefits. Learners are then able to practice what they learn though applied scenarios. Feedback is an important feature and integrated into all learning activities. 
Table 1. Course Overview

\begin{tabular}{|l|l|}
\hline \multicolumn{2}{|l|}{$\begin{array}{l}\text { This course is the first in a planned series on supervision that provides supervisors with an overview of key job responsibilities } \\
\text { and resources available to them to help improve job performance. }\end{array}$} \\
\hline Target audience: & This course is designed for newly appointed and veteran supervisors in the NYS workforce. \\
\hline Course structure: & $\begin{array}{l}\text { The course will consist of six topics focusing on key terminology and policies for attendance and } \\
\text { leave benefits, including: Introduction to Attendance and Leave Benefits, Attendance and Leave } \\
\text { Basics, Leave Categories, Other Types of Leave, The Supervisor's Role, and Conclusion and } \\
\text { Review. }\end{array}$ \\
\hline Course outcomes: & $\begin{array}{l}\text { This course will enable learners to gain knowledge of the rules concerning attendance and leave } \\
\text { benefits as outlined by state, agency, and federal policies and laws. Learners will use information } \\
\text { gained from the course to effectively apply attendance and leave benefits when working with their } \\
\text { employees. }\end{array}$ \\
\hline $\begin{array}{l}\text { Course objectives: } \\
\text { After completing this learners will } \\
\text { be able to: }\end{array}$ & $\begin{array}{l}\text { 1. Summarize the supervisor's role in enacting attendance and leave policies. } \\
\text { 2. Correctly apply attendance and leave policies in the workplace. } \\
\text { 3. Monitor and address employee attendance issues and concerns. } \\
\text { 4. Differentiate between the different types of leave and the appropriate use of each type of leave. } \\
\text { 5. Assist employees in using earned leave effectively. }\end{array}$ \\
\hline
\end{tabular}

The main menu introduces the course and provides the course overview. The guide for the course is used as a running character to help provide assistance with the course. After the introduction navigation buttons for each module appear on the page. Each module is designed with specific objectives related to the various content sections that are covered. Each section is designed to cover a specific area related to understanding attendance and leave benefits such as leave categories, leave benefits, types of leave, etc. A key objective for course participants is to gain a clear understanding of the supervisor's role and responsibilities related to time and attendance issues.

This is accomplished by prompting the learner to self- pace their course participation for content with a built- in review of materials and knowledge gain before proceeding to the next content section. All key points are graphically reinforced by the use of interaction icons and using scenario based activities that serve to engage the learner and keep them interested in the course content.

\section{Media Integration}

Graphically, the course uses images to illustrate activities and to represent terminology and topic icons. The media treatment for this course repeats for the other courses in all course offerings. This section provides screenshots of key course page designs and discusses key design features.

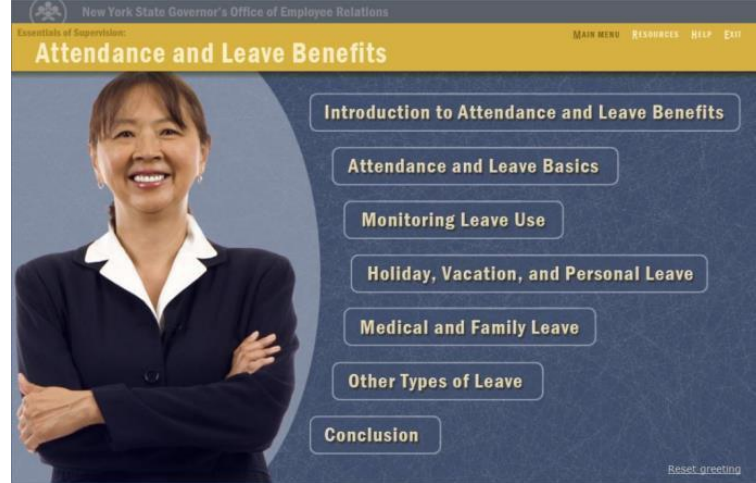

Figure 1. Course Home Page

The above Figure 1 illustrates the course home page. This page provides access to each of the topics and resources within the course. Learners will be able to return to the homepage at any point by using the navigation buttons located in the course header. Navigation Links: Course Header

- Home Page: Returns learners to the course home page. It will be inactive on the course homepage.

- Resources: Contains links to course resources. Links will open resources in a new window. Links to relevant resources will be made available during course topics for learners to reference.

- Help: Opens a page that provides assistance with the course.

- Exit: Closes the course. Learners who exit without completing the course will be able to return to the last page viewed via a button on the Main Menu.

The importance of easy to navigate media integration cannot be overstated. Experience has demonstrated that clunky navigation impedes learner outcomes and leads to frustration which can ultimately lead to learner dropout. 


\section{Effectiveness of Online Learning}

Evaluating the effectiveness of online delivery continues to be widely discussed, and in 2010, the United States Department of Education released Evaluation of Evidence-Based Practices in Online Learning: A Meta-Analysis and Review of Online Learning Studies. This report differed from prior studies of distance learning in that only web-based learning was included. Forty-five studies were analyzed across 50 effect sizes. It was found that, on average, students learning online perform modestly better than those receiving face-to-face instruction. Similarly, in a meta-analysis of job-related courses conducted by web-based instruction was found to be somewhat more effective $(6 \%)$ in teaching declarative knowledge than classroom instruction and equally effective in teaching procedural knowledge [16]. The results of the Sitzmann study were based on analysis of 96 studies and 71 effect sizes. These classroom and online learners reported equal satisfaction with their instructional experiences. Learners who self-selected online learning had better outcomes than learners who randomly assigned [16]. This is likely a consequence of level of comfort with technology and individual learning style. Other research further indicates that those self-selecting online courses tend to be more independent learners. Students that prefer classroom instruction tend to be more dependent and social [2]. A helpful approach to evaluating online learning programs is using the same Kirkpatrick evaluation model that is commonly used for classroom training [9]. However, it is also important to move beyond the Kirkpatrick levels of evaluation and include process evaluation as well as measuring for results. The focus should be on how to improve courses and that in the long run will make a better experience for learners. Process and results go hand-in-hand in achieving this outcome. Overall, there is a need for more rigorous evaluations to be conducted regarding the efficacy of e-learning for workplace training.

\section{Advantages of Online Learning}

Online learning has demonstrated that if used correctly it provides many benefits for both learners and employers. Advantages of online learning include consistent delivery of training to all participants, ability to offer just in time training to meet workforce needs and ability to deliver content in a manner that reduces information overload [4]. Learner control, practice and feedback have all been found to be effective means to improve learning outcomes. In courses where instructional methods were similar, online and classroom programs were evaluated as being equally effective. Where different instructional methods were used, online learning was found to be $11 \%$ more effective [16]. Training and development professionals must remain mindful that the same sound education principles that apply to classroom training need to be incorporated in elearning delivery as well.

\section{Measuring the Success of New York State's E-Learning Initiatives}

When comparing these findings regarding the effectiveness of New York State's e-learning courses the results are quite similar to the national research findings. Online training of the public workforce showed increased trainee understanding of the subject matter, and they had a favorable response to the use of online instructional methods. Using the mandatory Sexual Harassment course evaluations taken by state employees, PDP was able to demonstrate consistency with the national research regarding outcomes. The response rate of $36 \%$ to those surveyed was deemed to be valid for statistical analysis. The results are illustrated in Tables 2 and 3 shown as shown below. Table 2 provides evaluation results related to how well learners were able to understand the content. Table 3 refers to the instructional methods used such as games, quizzes, case studies and how well they were received by learners. In each case the data illustrates that the majority of participants were able to effectively understand the material and that the varied instructional approaches were considered to be a positive and effective way to present the material. Additional higher level evaluations aimed at obtaining more comprehensive analysis of the impact of training are under consideration for future courses. Overall, the evaluation results were encouraging and will be used in the development of future offerings. Paying attention to process evaluation is equally important as content evaluation. Both approaches provide a better overall picture of how well the course was received.

In a further review of evaluation data for online and classroom offerings by PDP for the course Infection Control which was delivered from 2006 up to 2011 similar findings are seen. The evaluation instrument used for the online deliveries differed slightly from the one used for the classroom workshops. However, the instruments have enough overlap that a general comparison of scores can be done. The evaluation framework is based on using the level one Kirkpatrick model [9].

The overall evaluation average for online infection control training was reported as 4.43 out of 5.00 based on 13 deliveries, and the overall evaluation average for classroom infection control training: 4.16 out of 5.00 based on 20 deliveries. Again, the findings re-enforce that e-learning delivery is as effective and in some cases can be more effective than classroom delivery of training. 
Table 2. Evaluation Results

The course increased my understanding of sexual harassment in the workplace and New York State's policies prohibiting such behavior.

\begin{tabular}{|l|l|l|l|l|l|}
\hline Times presented & 57626 & Times answered & 56682 & Min weight & 0 \\
\hline Max weight & 5 & Mean weight & 3.86 & Median weight & 4 \\
\hline
\end{tabular}

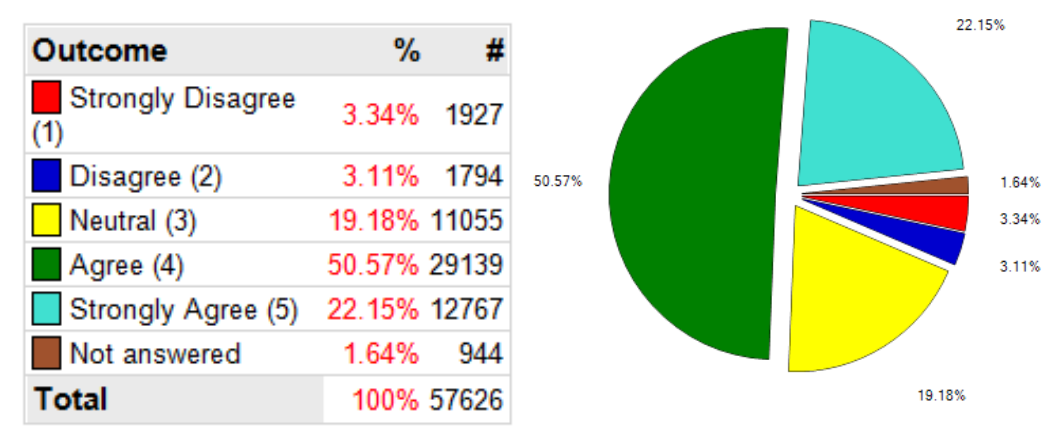

Table 3. Instructional Methods

The instructional methods (e.g., exercises, case studies, screen text, review questions, etc.) helped me understand the content.

\begin{tabular}{|l|l|l|l|l|l|}
\hline Times presented & 57626 & Times answered & 56577 & Min weight & 0 \\
\hline Max weight & 5 & Mean weight & 3.96 & Median weight & 4 \\
\hline
\end{tabular}

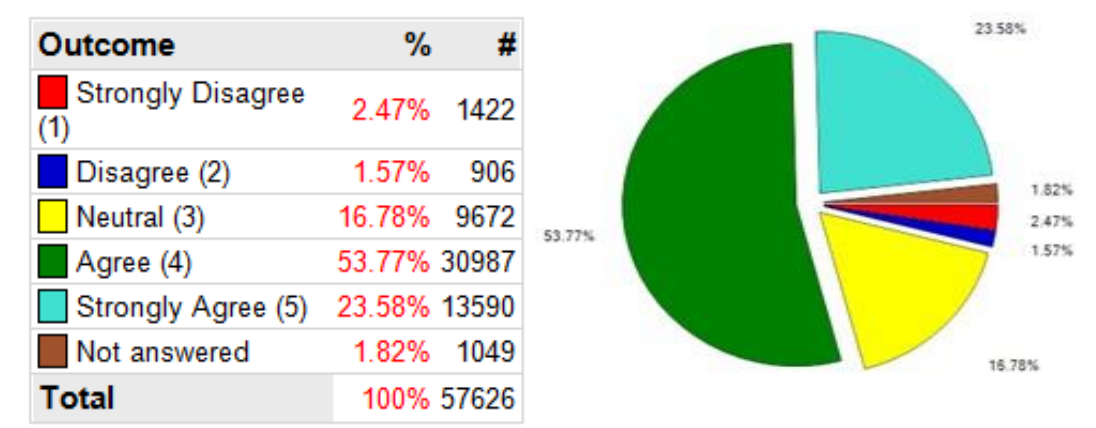

More comprehensive review and assessment still needs to be undertaken to validate findings and planning is underway to implement higher level evaluations.

\section{Conclusion}

Through this model program it was demonstrated that implementing evidenced based e-learning principles would produce the desired outcomes, and the proof of concept was therefore achieved. The technology based program validated that in a government work environment e-learning can be an effective instructional strategy for developing the knowledge, skills, and aptitudes that workers need in their state agency positions to ensure that the information and behaviors required to effectively carry out job duties and responsibilities in a uniform and consistent manner is achievable.

The preliminary research supports the principles that effective e-learning programs must consist of the following elements: sustainability, usefulness, and potential to return benefits to organizations as well as learners. To achieve these outcomes in an online learning format depend largely on how a course is designed, delivered, and ultimately evaluated. This model state program validates these presumptions and acts as a guide for successfully transitioning a 
course or program from a traditional classroombased approach to an e-learning delivery format. This model also illustrates the way in which a strong collaborative working relationship between higher education and state government can achieve the desired outcomes.

\section{References}

[1] Ardito, C et al, An Approach to Usability Evaluation of E-learning Applications, Universal Access in the Information Society, Vol. 4,No.3, .2006.

[2] Clark, R., Participant's Manual for How to Plan, Develop, and Evaluate Training 3rd edition, Cortez, Colorado, 2006. [3] Forester Research. (2009). The ROI of E-learning: A total economic impact. Retrieved September 12, 2013 from http://www.elearninglearning.com/

$\mathrm{html} / \mathrm{research} / \mathrm{roi} /$. [4] K. Guthrie, "Barriers to the Adoption of Online Learning in U.S. Higher Education", EDUCAUSE 47, no. 4, July/August 2002.

[5] Henry, P.,E-learning Technology, Content, and Services, Education and Training, Vol. 3, 920-940, 2001.

[6] Goldstein, I., and Ford, J., Training in Organizations: Needs Assessment, Development, and Evaluation, Wadsworth, California, 2002

[7] Internet Society (2012). Global Internet User Survey. Retrieved September 11, 2013 from http://www.internetsociety.org/publications.

[8] Kim, K., Bonk, C., Surveying the future of workplacee-learning the rise of blending, interactivity, and authentic learning Retrieved January 3, 2013 from https://elearnmag.acm.org/featured.cfm?aid=1073202.

[9] Kirkpatrick, D. L., Evaluating Training Programs, McGraw-Hill, New York, 1994.

[10] Lytle, R., On-line Education Continues to Grow, US News and World Report,

http://www.usnews.com/education/online-education/ articles/2011/11/11.

[11] Miller, L., 2012 State of the Industry, American Society for Training and Development, Alexandria, Virginia, 2012.

[12] New York State, The Redesign of State Government, Sage Commission, 2013.

[13] Nielsen, J., Usability 101, Introduction to Usability, Retrieved June 3, 2014, from http://www.nngroup.com/articles/usability-101introduction-to-usability/ .

[14] New York State Department of Civil Service. Workforce Reports. Retrieved January 11, 2013 from http://www.cs.ny.gov/businesssuite/Workforce-

Management/Succession-Planning/workforceplans.cfm.

[15] Ramage, Thomas R. (2002). The "No Significant Difference" phenomenon: A Literature Review. Dr. Thomas R. Ramage Scholarship. Paper 1. Retrieved September 17, 2013 from http://spark.parkland.edu/ ramage_pubs/1.

[16] Sitzmann, T., K. Kraiger, D. Stewart and R. Wisher, "The comparative effectiveness of web-based and classroom instruction: A meta-analysis", Personnel Psychology, 2006, pp. 59, 623-664.

[17] Steinmetz, L., Todd, First Line Management, Pennsylvania State University, Irwin Professional Publication, 1991.
[18] J. Strother, "An Assessment of the Effectiveness of elearning in Corporate Training Programs", The International Review of Research in Open and Distance learning 3, no. 1, April, 2002.

[19] United States Department of Education (2010). Evaluation of evidence-based practices in online learning: A meta-analysis and review of online learning studies. Retrieved September 17, 2013 from http://www2.ed.gov/ rschstat/eval/tech/evidence-based-practices/finalreport.pdf. [20] U.S. Department of Education Office of Planning, Evaluation, and Policy Development. Policy and Program Studies Service. (2010). Evaluation of Evidence-Based Practices in Online Learning: A Meta-Analysis and Review of Online Learning Studies.

[21] R. Wisher and T. Olson, "The Effectiveness of Webbased Training", U.S. Army Research Institute for the Behavioral and Social Sciences, January, 2003.

[22] E. Welch, C. Wanberg, K. Brown, and M. Simmering, "E-learning: Emerging uses, empirical results and future directions", International Journal of Training and Development 7, no.4, 2003, pp. 245-258. 